\title{
CONCERNING GRIBOV VACUUM COPIES IN NON-ABELIAN GAUGE THEORY *
}

Paul H. Frampton, William F. Palmer and Stephen S. Pinsky

Department of Physics

The Ohio State University

Columbus, Ohio 43210

Abstract

Construction of gauge field configurations $\mathrm{A}_{\mu}^{a}(\mathrm{x})$ in an SU(2) Yang-Mills theory satisfying everywhere $F_{\mu \nu}^{a}(x)=0$ is discussed. Using the method of sections, a field related to the zero-size limit of an instanton is presented. The corresponding limit for a multi-instanton solution requires a generalization of the Landau gauge condition. Finally, an alternative method and explicit solution is given for the case of $\partial_{\mu} A_{\mu}^{a}=0$.

* Supported in part by the U.S. Department of Energy under contract number EY-76-C-02-1545.*000. 


\section{DISCLAIMER}

This report was prepared as an account of work sponsored by an agency of the United States Government. Neither the United States Government nor any agency Thereof, nor any of their employees, makes any warranty, express or implied, or assumes any legal liability or responsibility for the accuracy, completeness, or usefulness of any information, apparatus, product, or process disclosed, or represents that its use would not infringe privately owned rights. Reference herein to any specific commercial product, process, or service by trade name, trademark, manufacturer, or otherwise does not necessarily constitute or imply its endorsement, recommendation, or favoring by the United States Government or any agency thereof. The views and opinions of authors expressed herein do not necessarily state or reflect those of the United States Government or any agency thereof. 


\section{DISCLAIMER}

Portions of this document may be illegible in electronic image products. Images are produced from the best available original document. 


\section{INTRODUCTION}

A new ingredient in the quantization of non-abelian gauge theories, and a potentially important step towards their physical interpretation, has been pointed out recently by Gribov. ${ }^{1}$ The principal idea is that a choice of gauge, such as the coulomb gauge, leaves a non-trivial residual freedom in the choice of gauge field, this extra freedom being absent in the case of electrodynamics. For example, it is possible to find gauge transforms of the pure vacuum such that the Yang-Mills field

$$
F_{\mu \nu}^{a}=\partial_{\mu} A_{\nu}^{a}-\partial_{\nu} A_{\mu}^{a}+g \varepsilon^{a b c} A_{\mu}^{b} A_{\nu}^{c}
$$

vanishes everywhere and such that the local gauge condition is preserved. In what follows, we refer to such $\mathrm{A}_{\mu}^{a}$ as vacuum copies.

It has been speculated that this copying phenomenon may be of fundamental importance in the physical implications of Yang-Mills theory, perhaps: pertaining to the elusive property of confinement. We leave this question open, but the possibility provides a strong motivation for this work; indeed, the literature on this subject is already expanding rapidly. ${ }^{2}$

In Ref.1, the discussion centered on the Coulomb gauge which served only as an example. Here we shall explore vacuum copies in the Landau gauge and a generalization thereof. The principal difference is that coulomb gauge copies resemble the Wu-Yang form ${ }^{3}$ while those of the Landau gauge are expected to have possible structures related to the instanton of Belavin et a1. 4

To construct these copies we initially break translational invariance, although this would be restored at the end in any realistic calculation, using e.g. the method of collective coordinates. In Sec.II we consider the zero-size limit of ọne instanton, centered around one preferred space-time point and use the method of sections to construct a copy. In Sec.III, we study the zero-size limit of the muiti-instanton, and find that the Landau 
condition must be generalized, for this case.

In Sec.IV we present an alternative approach to vacuum copies in the Landau gauge, together with an explicit solution. Finally, in Sec.V are the concluding remarks, including a brief comment on the Faddeev-Popov 5 formalism.

\section{ZERO-SIZE LIMIT OF ONE INSTANTON}

It appears that to maintain any given gauge condition and to find a vacuum beyond the pure case $A_{\mu}^{a}=0$ it is necessary to break translational invariance. Thus, we consider first a vacuum copy with one preferred spacetime center. Full translational invariance can then be restored by averaging all possible locations of this center.

For the Landau gauge. we start from the BPST instanton ${ }^{4}$ in Euclidean space given by

$$
\begin{aligned}
\mathrm{A}_{\mu}^{\mathrm{a}} & =\frac{2}{\mathrm{~g}} \frac{\eta_{\mu \nu}^{a} \mathrm{x}_{\nu}}{\left(\mathrm{x}^{2}+\lambda^{2}\right)} \\
\mathrm{F}_{\mu \nu}^{\mathrm{a}} & =-\frac{4}{\mathrm{~g}} \eta_{\mu \nu}^{\mathrm{a}}\left(\frac{\lambda}{\mathrm{x}^{2}+\lambda^{2}}\right)^{2} .
\end{aligned}
$$

As is well known ${ }^{4}$ this instanton carries a topological charge given by

$$
\begin{aligned}
Q & =\frac{g^{2}}{32 \pi^{2}} \int d^{4} \times F_{\mu \nu}^{a} \tilde{F}_{\mu \nu}^{a} \\
& =1
\end{aligned}
$$

Here $\widetilde{F}_{\mu \nu}^{a}=\frac{1}{2} \epsilon_{\mu \nu \alpha \beta} \hat{F}_{\alpha \beta}$. Using Gauss' theorem, this may be-re-written

$$
Q=\frac{g^{2}}{32 \pi^{2}} \int_{x^{2} \rightarrow \infty} d^{3} \sigma_{\alpha} T_{\alpha}
$$

where 


$$
T_{\mu}=\varepsilon_{\mu \nu \alpha \beta}\left[2 A_{\nu}^{a} \partial_{\alpha} A_{\beta}^{a}+\frac{2}{3} g e^{a b c} A_{\nu}^{a} A_{\alpha}^{b} A_{\beta}^{c}\right]
$$

The parameter $\lambda$ in Eq.(2) is arbitrary and reflects the scale-invariance of the theory; it characterizes the size of the instanton. For general $\lambda$ one has

$$
\begin{aligned}
T_{\mu} & =\frac{16 x_{\mu}}{g^{2}\left(x^{2}+\lambda^{2}\right)^{3}}\left(x^{2}+3 \lambda^{2}\right) \\
\partial_{\mu} T_{\mu} & =\frac{192}{g^{2}}\left(\frac{\lambda}{x^{2}+\lambda^{2}}\right)^{4}
\end{aligned}
$$

In the zero-size limit $\lambda \rightarrow 0$, we have $\mathrm{F}_{\mu \nu^{3}}^{\mathrm{a}}=0$ for all $\mathrm{x}_{\mu}$ except at the origin, where a singularity develops. This singularity is where the topological charge accumulates since one now has

$$
\mathrm{F}_{\mu \nu}^{\mathrm{a}} \tilde{\mathrm{F}}_{\mu \nu}^{\mathrm{a}}=\frac{32 \pi^{2}}{\mathrm{~g}^{2}} \delta^{4}(\mathrm{x})
$$

Now, for a vacuum copy, defined by $F_{\mu \nu}^{a}=0$ everywhere, it is clear from Eq. (4) that the net topological charge Q must vanish. Thus, the zero-size limit of the instanton is not a vacuum copy, as it stands.

To clarify completely the topological properties it is instructive to make the singular gauge transformation

$$
U(x)=\left(x_{4}-i \underline{\sigma} \cdot \underline{x}\right) / \sqrt{x^{2}}
$$

to arrive at the so-called "singular" gauge ${ }^{6}$

$$
\begin{aligned}
& \hat{A}_{\mu}=U^{\dagger} \dot{A}_{\mu} U-\frac{i}{g} U^{\dagger} \partial_{\mu} U \\
& \hat{A}_{\mu}^{a}=\frac{\lambda^{2}}{x^{2}} A_{\mu}^{a}
\end{aligned}
$$

with $A_{\mu}^{a}$ given by Eq. (2). This is now singular at the origin for all $\dot{\lambda} \neq 0$ 
and correspondingly falls away too quickly at large distance to sustain a topological charge. From Eq. (13) one checks that

$$
\begin{aligned}
& \hat{\mathrm{T}}_{\mu}=-\frac{16 \lambda^{4} x_{\mu}}{g^{2}\left(x^{2}+\lambda^{2}\right)^{3} x^{4}} \\
& \hat{F}_{\mu \nu}^{a} \tilde{\hat{F}}_{\mu \nu}^{a}=\partial_{\mu} \hat{T}_{\mu} \\
& \quad=\frac{192}{g^{2}}\left(\frac{\lambda}{x^{2}+\lambda^{2}}\right)-\frac{32 \pi^{2}}{g^{2}} \delta^{4}(x) .
\end{aligned}
$$

The crucial point is that now the topological charge is $\hat{Q}=0$ for all $\lambda$. That is, Q defined by Eq. (4) is not gauge invariant under singular transformations.

A vacuum copy should be such that all truly gauge invariant quantities coincide with their values for the pure vacuum $\mathrm{A}_{\mu}^{\mathrm{a}}=0$.

Such a copy may be defined from Eqs. (2) and (13) by using the method of sections. $^{7}$ We therefore define $A_{\mu}^{a}(x)$ by

$$
A_{\mu}^{a}(x)= \begin{cases}\lim _{\lambda \rightarrow 0}\left(A_{\mu}^{a}\right)=\frac{2}{g} \frac{\eta_{\mu \nu}^{a} x_{\nu}}{x^{2}} & x^{2}>\varepsilon^{2} \\ \lim _{\lambda \rightarrow 0}\left(\hat{A}_{\mu}^{a}\right)=0 . & x^{2}<\varepsilon^{2}\end{cases}
$$

Here $\varepsilon^{2}$ may be made arbitrarily. small but non-zero. At the boundary between the two regions, the two functions are related by the gauge transformation Eq. (11) which is regular where it is used.

\section{ZERO-SIZE LIMIT OF MULTI-INSTANTON}

Although an explicit formula for the most general $N$ instanton configuration is not at hand, 't Hooft ${ }^{6}$ has provided one with $5 \mathrm{~N}$ parameters (rather than the allowed $8 \mathrm{~N}-3$ ) that is sufficiently general for our purpose. It is given by 


$$
A_{\mu}^{a}=\frac{1}{g} \eta_{\mu \nu}^{a} \partial_{\nu} \ln \left(1+\sum_{i=1}^{N} \frac{\lambda_{i}^{2}}{x_{i}^{2}}\right)
$$

in which $x_{i \mu}=\left(x-x_{i}\right)_{\mu}$ and where $x_{i \mu}, \lambda_{i}$ are the location, size respectively of the ith instanton.

This formula is written in the singular gauge $A_{\mu}^{a} \sim x^{-3}, T_{\mu} \sim x^{-7}$ and hence has net topological charge $Q=0$. In the zero-size limit, it becomes the pure vacuum $A_{\mu}^{a}=0$.

Thus, to investigate such vacuum copies it is essential to transform to a non-singular gauge. For the case $\dot{\mathrm{N}}=2$ the appropriate singular gauge transformation is given by

$$
\begin{aligned}
& U(x)=\left(\hat{z}_{4}(x)+i \underline{\sigma} \cdot \underline{\hat{z}}(x)\right) \\
& z_{\mu}(x)=\alpha \frac{x_{1 \mu}}{x_{1}^{2}}+\beta \frac{x_{2 \mu}}{x_{2}^{2}}
\end{aligned}
$$

with $\hat{z}_{\mu}=z_{\mu} / \sqrt{z^{2}}$.

Actually, one needs $\alpha=-\beta$ but we have considered the more general case where $\alpha, \beta$ are any real constants. For the zero-size limit one then has

$$
\begin{array}{r}
A_{\mu}^{a}=\frac{2}{g} \eta_{\alpha \beta}^{a}\left(\partial_{\mu} \hat{z}_{\alpha}\right) \hat{z}_{\beta} \\
\partial_{\mu} A_{\mu}^{a}=\frac{2}{g} \eta_{\alpha \beta}^{a}\left(\square \hat{z}_{\alpha}\right) \hat{z}_{\beta}
\end{array}
$$

After some algebra this gives

$$
\partial_{\mu} A_{\mu}^{a}=-\frac{8 \alpha \beta}{g x_{1}^{4} x_{2}^{4}} \frac{1}{z^{2}}\left(x_{2}^{2}-x_{1}^{2}\right) \eta_{k \lambda}^{a} x_{1 k} x_{2 \lambda}
$$

and the right-hand side of (23) is non-vanishing for all $\alpha, \beta \neq 0$. If we specialize to the zero-size limit of the two instanton solution $(\alpha=-\beta)$ one finds ${ }^{8}$ 


$$
A_{\mu}^{a}=\frac{2}{g\left(x_{1}-x_{2}\right)^{2}}\left[\eta_{\mu \nu}^{a}\left(\frac{x_{1 \nu}}{x_{1}^{2}}-\frac{x_{2 \nu}}{x_{2}^{2}}\right)\left(x_{2}^{2}-x_{1}^{2}\right)-2\left(\frac{x_{1 \mu}}{x_{1}^{2}}-\frac{x_{2 \mu}}{x_{2}^{2}}\right) \eta_{k \lambda}^{a} x_{1 k} x_{2 \lambda}\right] .
$$

It is easily checked that the divergence now satisfies Eq. (23).

Thus, it is possible to construct vacuum copies from such zero-size limits, using the method of sections, if one generalizes the Landau condition to

$$
\partial_{\mu} A_{\mu}^{a}=\sum_{\substack{i, j=1 \\ i<j}}^{N} c_{i j}(x) \eta_{k \lambda}^{a} x_{i k} x_{j \lambda}
$$

where $\mathrm{N}$ is the number of instantons. Only in the case $\mathrm{N}=1$ does the right-hand side of (25) vanish, thus restoring the Landau gauge as was discussed in the previous sub-section.

We now turn to an alternative approach in the Landau gauge.

\section{ALTERNATIVE APPROACH IN LANDAU GAUGE}

For the general gauge transformation of the form, Eq. (19) above, the field $A_{\mu}^{a}$ is given by Eq. (21) and hence its divergence by Eq. (22). In order to satisfy the Landau condition, therefore, we need

$$
\eta_{\mu \nu}^{a}\left(\square \hat{z}_{\mu}\right) \hat{z}_{\nu}=0
$$

together with

$$
\hat{z}_{\mu} \hat{z}_{\mu}:=1
$$

in order that the gauge transformation $U$ be unitary and unimodular $\left(\hat{z}_{\mu}=\right.$ real). To fulfill the nonlinear constraint, Eq.(27), we parametrize

$$
\hat{z}_{\mu}=\left(\begin{array}{l}
\cos \phi \sin \theta \sin \alpha \\
\sin \phi \sin \theta \sin \alpha \\
\cos \theta \sin \alpha \\
\cos \alpha
\end{array}\right)
$$


where the three functions $\alpha(x), \theta(x), \phi(x)$ are constrained only by Eq. (26). The necessary and sufficient condition to satisfy Eq.(26) is the generalized Klein-Gordon-type equation

$$
(\boldsymbol{\nabla}+\beta(\mathrm{x})) \hat{z}_{\mu}=0 \quad \mu=1,2,3,4
$$

with $\beta(x)$ an arbitrary function, independent of the choice of $\mu$.

To find an explicit solution, we may take as an example $\theta=\frac{\pi}{2}$ whereupon Eq. (29) gives just two equations

$$
\begin{aligned}
& \alpha=\left(\partial_{\mu} \phi\right)^{2} \sin \alpha \cos \alpha \\
& \phi=-2 \cos \alpha \partial_{\mu} \phi \partial_{\mu} \alpha .
\end{aligned}
$$

We may satisfy Eq. (3I) by the ansatz

$$
\begin{aligned}
& \varnothing=\mathrm{Ax}_{4}+\mathrm{B} \\
& \alpha=\alpha\left(\mathrm{x}_{1}, \mathrm{x}_{2}, \mathrm{x}_{3}\right) .
\end{aligned}
$$

Defining $s=\hat{c}_{i} x_{i}$ where $\hat{c}_{i}$ is an arbitrary unit vector, Eq. (30) may be re-written

$$
\nabla^{2}(2 \alpha)=\frac{d^{2}}{d s^{2}}(2 \alpha)=A^{2} \sin 2 \alpha .
$$

This is an equation familiar from Sine-Gordon theory, or equivalently from the undamped pendulum, and there is a solution

$$
\tan \frac{\alpha}{2}=e^{ \pm A s}
$$

Substituting Eqs.(32) and (35) into Eqs.(21) and (28) we find

$$
\begin{aligned}
& A_{4}^{a}=\frac{2 A \sin \alpha}{g}\left(-\cos \alpha \sin \left(x_{4}+B\right), \cos \alpha \cos \left(A x_{4}+B\right), \sin \alpha\right) \\
& \underline{A}^{a}=\frac{2 A \underline{\hat{c} \sin \alpha}}{g}\left(\cos \left(A x_{4}+B\right), \sin \left(A x_{4}+B\right), 0\right)
\end{aligned}
$$


where the components $a=(1,2,3)$ are written explicitly.

For a suitable re-definition of the coordinate axes $\mu=1,2,3,4$, this field $A_{\mu}^{a}$ satisfies also the Coulomb gauge $\underline{\nabla} \cdot \underline{A}^{a}=0$; it is then a vacuum copy which avoids the spherical-symmetry assumptions made in Ref.1.

\section{CONCLUDING REMARKS}

Given the existence of vacuum copies, one must next decide how to incorporate them into calculations of physical quantities.

The most natural formalism is that of Faddeev and Popov, ${ }^{5}$ suitably modified. The original version may be written symbolically for a vacuumvacuum amplitude

$$
\int d A_{\mu} \delta\left(\partial_{\mu} A_{\mu}\right) \Delta(A) e^{i S}
$$

One method of modification is to write ${ }^{9}$

$$
\int D A_{\mu} \delta\left(\partial_{\mu} A_{\mu}\right)\left(\sum_{n} \Delta\left(A^{(n)}\right)^{-1}\right)^{-1} e^{i S}
$$

where $\Delta\left(A^{(n)}\right)$ is the jacobian evaluated for the $n^{\text {th }}$ copy. An equivalent prescription is easily. seen to be

$$
\int d A_{\mu} \frac{\delta\left(\partial_{\mu} A_{\mu}\right) \Delta(A)}{N(A)} e^{i S}
$$

where $N(A)$ is a functional giving the number of copies for a prescribed $A_{\mu}^{a}(x)$. This alternative form may be more useful for practical calculations. 


\section{REFERENCES}

1. V. N. Gribov, Lecture at the 12 th Winter School of the Leningrad Nuclear Physics Institute, 1977. SLAC-TRANS-176 (1977).

2. A partial list of articles wholly or partly devoted to discussions of the points raised in Ref.1 follows: C. M. Bender, T. Eguchi and H. Pagels, Rockefeller University preprint (1977); R. Jackiw, J. Muzinich and C. Rebbi, Brookhaven Laboratory preprint (I977); J. Goldstone and R. Jackiw, M.I.T. preprint (1978); H. D. I. Abarbane1 and J. Barte1s, Fermilab preprint (1977); S. Sciuto, Phys. Lett. $\underline{71 B}, 129$ (1977); M. Ademollo, E. Napolitano and S. Sciuto, CERN preprint (1977); L. F. Abbott and T. Eguchi, Phys. Lett. $\underline{72 B}$; 215 (1977); M. B. Halpern and J. Koplik, U.C. Berkeley preprint (1977);

D. Amati and A. Rouet, Phys. Lett. $\underline{73 B}, 39$ (1978); R. D. Peccei, Stanford Univ. preprint (1977); Y. Iwasaki, Princeton preprint (1978); A. P. Balachandran et a1., Phys. Rev. Lett. 40, 988 (1977); etc.

3. T. T. Wu and C. N. Yang, in Properties of Matter under Unusual Conditions, edited by H. Mark and S. Fernbach (Interscience, New York, 1969), pp.349-354; see also G. 't Hooft, Nuc1. Phys. B79, 276 (1974), A. M. Polyakov, JETP Letters $\underline{20}, 194$ (1974).

4. A. A. Belavin, A. M. Polyakov, A. S. Schwartz and Yu. S. Tyupkin, Phys. Lett. $\underline{59 B}, 85$ (1975).

5. V. N. Popov and L. D. Faddeev, Kiev Report ITP 67-36 (1967), translated as Fermilab. report NAL-THY-57 (1972); L. D. Faddeev and V. N. Popov, Phys. Lett. 25B, 29 (1967); L. D.'Faddeev, Theor. and Math. Phys. 1, 3 (1969); see also E. S. Fraokin and I. V. Tyutin, Phys. Rev. D2, 2841 (1970); I.'B. Khrip1ovich, Sov. J. Nuc1. Phys. 10, 235 (1970). For a review, see e.g. P. H. Frampton, UCLA preprint UCLA/77/TEP/21 (1977).

6. G. 't Hooft, unpublished and Cora1 Gables Conference, January 1977; see also R. Jackiw, C. Nohl and C. Rebbi, Phys. Kev. D 15, 1642 (1977). 
$\cdot \quad \cdot \quad \cdot \quad \cdot$

11

7. T. T. Wu and C. N. Yang, Nucl. Phys. B107, 365 (1976).

8. J. J. Giambiagi and K. D. Rothe, CERN preprint (1977).

9. See, for example, C. M. Bender et a1., Ref.2. 